\title{
Schmidt Telescopes: Their Past, Present and Future
}

\author{
Russell D. Cannon \\ Anglo-Australian Observatory, PO Box 296, Epping, NSW 2121
}

My objective here is to give a brief overview of Schmidt telescope astronomy, to explain why we decided to organise this Symposium and to outline what it is that we hope to achieve during the meeting.

\section{The principle of the Schmidt telescope}

With such a gathering of experts in the field it hardly seems necessary to define the subject of this meeting, but for the benefit of non-specialists let me briefly outline the history of Schmidt telescopes and their use in astronomy. The basic principle of the Schmidt telescope was discovered or invented, depending on your point of view, by our eponymous hero, Bernhard Schmidt, in about 1930. Most conventional large optical telescopes have a rather small field of view, measured in minutes of arc. Working at the Hamburg Observatory, Schmidt realised that by placing an aspheric corrector lens as an aperture stop at the centre of curvature of a spherical mirror, it should be possible to build a telescope with a field of view measured in degrees. He built a small prototype to demonstrate his idea and astronomers immediately realised its potential for carrying out large-scale photographic surveys of the sky.

\section{Development of Schmidt astronomy}

The first moderately large Schmidt telescopes were those built in Hamburg and at Palomar shortly before the Second World War, followed in 1948 by the famous Palomar 48-inch (1.2 m) Schmidt (subsequently renamed the Oschin Schmidt) and then by a spate of small and middle-sized Schmidts built during the 1950's and 1960's in many different countries. Construction of large Schmidt telescopes, with apertures of $1.0 \mathrm{~m}$ or greater, proceeded more uniformly with a total of eight being built between 1945 and 1980. Almost no new Schmidt telescopes have been built during the last 15 years although a few older ones have been moved to better astronomical sites.

\section{The major sky surveys}

Three of the world's largest Schmidt telescopes were built with the specific intention of using them to carry out systematic surveys of the sky, in order to 
locate targets for large optical telescopes being built at the same time. This pattern was set by the construction of the 48-inch Schmidt alongside the Hale 200-inch (5 m) telescope on Palomar Mountain. The National Geographic Society - Palomar Observatory northern sky survey, carried out between 1950 and 1958, has turned out to be an immensely influential fundamental database for all astronomy. A mosaic of almost 1000 pairs of blue and red photographs covered the entire sky accessible from California, from the north pole down to $-33^{\circ}$ declination. The resulting Sky Atlas is available worldwide in most astronomical libraries. Originally conceived simply as a tool for finding interesting stars, nebulae and galaxies for further study with the 200-inch and other large optical telescopes, the Palomar survey has of course turned out to be of immense value in the identification of objects found at other wavelengths, first radio sources and more recently $\mathrm{X}$-ray, ultraviolet and infrared sources. The Schmidt data have also turned out to be invaluable in their own right, for example in studies of the large scale structure of the universe. The success of this northern survey led the builders of the large southern telescopes in the 1970's to erect companion Schmidt telescopes, the UK $1.2 \mathrm{~m}$ Schmidt Telescope alongside the $3.9 \mathrm{~m}$ AngloAustralian Telescope in Australia and the ESO $1.0 \mathrm{~m}$ Schmidt beside the $3.6 \mathrm{~m}$ in Chile. These two southern Schmidts carried out the ESO-SERC survey of the southern sky, between 1974 and 1990. Given the advances in photographic emulsions and telescope technology over the intervening 20 years, the ESOSERC survey reached about 1.5 magnitudes fainter than the earlier Palomar survey and led to the repeat of the northern survey which is currently under way.

\section{Schmidt telescopes as data gatherers}

Besides their obvious use as survey telescopes, the great feature of Schmidts is their effectiveness as data-gatherers. Purely in terms of information theory, a Schmidt telescope out-performs any other of the same aperture by an enormous factor. This has meant somewhat paradoxically that many of the world's Schmidts have been built at relatively poor or mediocre sites, but the justification is that if you have only a small proportion of clear nights each year, you had better make the best use of them that you can. Similarly, one Schmidt telescope can support many astronomers in a diverse range of research, so a Schmidt was often the natural choice where the resources for building telescopes were limited.

\section{The era of quantitative data}

A single photograph from one of the large Schmidts contains about a billion pixels; more practically, each deep exposure records between $10^{5}$ and $10^{6}$ images of stars and galaxies. This limited early work to visual inspection of the atlas prints, or to detailed study of very small regions. To exploit the power of the Schmidts properly required the development of very fast automatic plate measuring machines, such as COSMOS in Edinburgh, the APM in Cambridge and the special PDS machines at the Space Telescope Science Institute. A related problem was to calibrate the intrinsically non-linear photographic data; this was beyond the reach of single-object photoelectric photometry but can now 
be achieved quite easily using CCDs on moderate size telescopes. As a result, Schmidt telescopes can now be used as primary research telescopes in their own right for a multitude of projects, ranging from the study of the universe on the largest scales, by mapping out the distribution of quasars and galaxies, to a census of asteroids in our immediate vicinity in the solar system. Other examples include delineating the structure of the Milky Way Galaxy and its nearest neighbours, the Magellanic Clouds; searches for unseen dark matter through stellar variability due to gravitational lensing; and finding nearby 'brown dwarf' stars through their colours and rapid apparent motions. Another consequence of the advent of the measuring machines is that the Schmidt sky surveys are becoming widely available in digitised form. No longer is it necessary to go to a film library and pore over the pictures through a microscope; now the surveys are widely available on CD ROM and soon anyone with access to a good graphics computer terminal and the Internet will be able to display any part of the sky at will. More than that, it is becoming trivial to overlay optical pictures of the sky with maps obtained at other wavelengths.

\section{Promising developments}

Most photographic emulsions detect at best only a few per cent of incoming photons. As a result photography has been superseded for most astronomical applications by the use of electronic detectors such as CCDs, with peak efficiencies now approaching 100 per cent. However, photography remains the only way to cover the full field of a Schmidt without any loss in resolution; in this application the huge number of photographic 'pixels' still outweighs the much higher quantum efficiency of CCDs. It has recently been realised that Kodak Tech Pan film can give efficiencies which probably exceed 10 per cent, while the measuring machines permit the digital addition of data from a series of identical exposures. Combining these two techniques means that photographic surveys can now go two or more magnitudes deeper than the best available up to now, and at higher spatial resolution, giving results which are unlikely to be matched by CCDs over large areas of sky for some time to come. However, for other applications the era of CCDs in Schmidt telescopes has already begun, since it is easy to cover fields of up to a degree with a single exposure. A third quite separate development has been the use of optical fibres, pioneered in the UK Schmidt, to observe around 100 faint stars or galaxies simultaneously by feeding their light to an external spectrograph.

\section{Current limitations}

One concern is the rapidly decreasing availability of glass photographic plates. Fortunately, emulsions on film are a satisfactory alternative, in fact they are much cheaper and in some cases better than the old glass plates. And although single CCDs cannot compete with photography in Schmidt telescopes, several groups are developing arrays of CCDs which will eventually supersede photography. The questions which have to be addressed include: what should the large Schmidt telescopes do as the current all-sky surveys near completion, and what 
are the best scientific programmes for the smaller Schmidts, many of which are now limited by rapidly increasing levels of artificial light?

\section{Future options}

There are two classes of project here: those which have been feasible on the large Schmidts for many years, but which have not been fully explored because little telescope time was available while the sky surveys were in progress; and those which have only recently become feasible as a result of technological advances such as digital addition of exposures and the use of CCDs or optical fibres. Examples in the former category include: systematic sky surveys with objective prisms, to find quasars and other rare classes of object; parallax surveys for nearby stars, using appropriate time series of exposures; deep proper motion studies in selected Milky Way fields, to study Galactic kinematics; searches for quasars and various classes of variable stars from long series of exposures; and comprehensive asteroid searches.

\section{Immediate objectives}

One aim of this meeting is to review the past and current uses of Schmidt telescopes and to explore the scientific and technological options for Schmidt astronomy in future, and to see what are the best ways of utilising existing facilities and what cases can be made for the building of new facilities. Another parallel objective is to explore the options for international cooperation, particularly here in the Asian-Pacific region, in the use of Schmidt telescopes and the distribution of digitised data. 